\title{
Effective Field Theory of Electron Motion in the Presence of Random Magnetic Flux
}

\author{
Shou-Cheng Zhang \\ IBM Research Division, Almaden Research Center, San Jose, CA 95120 \\ and \\ Department of Physics, Stanford University, Palo Alto, CA 94305 \\ Daniel P. Arovas \\ Department of Physics, UC at San Diego, La Jolla, CA 92093
}

(September 17, 2018)

\begin{abstract}
We construct a nonlinear $\sigma$ model to describe a system of non-interacting electrons propagating in the presence of random magnetic flux. We find a term describing the long ranged logarithmic interaction between the topological density of the non-linear sigma model, and argue that this could give rise to a Kosterlitz-Thouless transition from the localized phase to a phase with power law correlations and continuously varying conductances. We provide a physical interpretation of our results in terms of the scattering of edge states of the magnetic domains in different regions.
\end{abstract}

PACS numbers: $7.10 . \mathrm{Bg}, 71.55 . \mathrm{Jv}$

The localization problem in two dimensions has been investigated extensively in the past twenty years [1]. Recently there has been considerable interest in studying the problem of noninteracting two-dimensional electrons propagating in the presence of random magnetic flux. Physically this problem arises in the study of the quantum Hall systems near even denominator filling fractions 2. 2 and could also be related to holon propagation in chiral spin liquid [5] and the gauge theory describing hole motion in an antiferromagnet [6]. Single particle properties, such as the one-body Green's function and the density of states, have been addressed [7 10]. A more difficult question to assess is that of localization. Naïvely, one would assume that the conventional unitary class sigma model description holds, in which case the beta function vanishes to the first order, but is non-vanishing and negative to the second order [11, indicating weak localization. For the random flux tight binding model,

$$
H=-t_{0} \sum_{\langle i j\rangle} e^{i a_{i j}}|i\rangle\left\langle j\left|+e^{-i a_{i j}}\right| j\right\rangle\langle i|
$$

with the phase $e^{i a_{i j}}$ on each link uniformly distributed on the unit circle, numerical calculations in squares and long strip geometries are consistent with the fact that states are localized near the bottom of the band [12,13, , 1 . However, as the energy approaches the band center, the localization length increases rapidly, and the interpretation of numerical data becomes problematic. In refs. [2, 13, 4] it is was argued that states near the band center are extended, whereas ref. [12] argues that all states are localized. In view of the rapid increase of the localization length and the limit of sample sizes in numerical calculations, it is important to develop analytical methods to address the issue of localization.

In this work, we construct a field theory to describe the random flux model. We envision a system with both random magnetic flux in addition to a random scalar potential. Our starting point is the non-linear sigma model of the unitary ensemble which is appropriate for the present case with broken time reversal invariance. In the lattice model defined by equation (1), the random flux is correlated only over the range of an elementary plaquette. However, for the field theory formulation it is more convenient to consider the opposite limit where the correlation length of the random magnetic field is large compared to the elastic mean free path. While these two models addresse two different limits, we hope that the basic physics continuously interpolates between them. The basic degrees of freedom of the field theory model are the matrices $Q$ defined on the coset space of $U(2 m) / U(m) \times U(m)$; in the localization problem we are interested in the limit of vanishing $m$. One remarkable feature of this field theory is the existence of the topological excitation called instantons. It was argued in ref. [14] that in the presence of a uniform magnetic field, there is a term describing the coupling of the Hall conductance to the instanton density. This topological term is responsible for the existence of extended states necessary for the integer quantum Hall effect. In the case of random magnetic flux with no net average, such a topological term can not exist. However, it is perfectly sensible to think of a "local topological angle" associated with a given magnetic domain, which is coupled to the local instanton density. We are able to demonstrate that there is a finite stiffness to changes this local topological angle. This leads to an effective long ranged logarithmic interaction between the instanton density. We further argue that this long ranged interaction could lead to a Kosterlitz-Thouless transition between the free and confined phase of the instantons. In the former phase all states are localized whereas extended states exist in the later case and there is gapless 
excitations in the system which gives power law correlations.

Physically, one can think of these gapless excitations as the edge states of the fluctuating magnetic domains. These extended edge states can exist over a distance larger than the localization length, just as in the case of the quantum Hall effect. These edge states are confined in the one dimensional network where the magnetic field changes sign. Since the magnetic field is randomly distributed with a zero average, this network always percolate the entire system. Of extreme importance is the scattering of the edge states with different sense of rotation, or different chirality. In the phase where this scattering is relevant, a mass gap opens up and all states are localized. In the phase where the scattering is irrelevant, the edge states remain gapless and carry currents. There are power law correlations and a finite, continuously varying conductance.

We begin with the non-linear sigma model derived by Levine, Libby and Pruisken [14] for the case of electrons propagating in a random scalar potential and an uniform magnetic field. The Lagrangian takes the following form:

$$
\mathcal{L}=\frac{1}{8} \sigma_{x x} \operatorname{Tr}\left(\partial_{\mu} Q\right)^{2}-\frac{1}{8} \sigma_{x y} \operatorname{Tr} \epsilon^{\mu \nu} Q \partial_{\mu} Q \partial_{\nu} Q
$$

where $Q$ is a matrix defined on the coset space of $U(2 m) / U(m) \times U(m)$, and $\sigma_{x x}$ and $\sigma_{x y}$ are the longitudinal and the Hall conductances respectively, defined as

$$
\begin{aligned}
& \sigma_{x x}=\left\langle\hat{\sigma}_{x x}\right\rangle=-\left\langle\operatorname{Tr} \Pi_{x}\left(G^{+}-G^{-}\right) \Pi_{x}\left(G^{+}-G^{-}\right)\right\rangle_{\mathrm{imp}} \\
& \sigma_{x y}=\sigma_{x y}^{\mathrm{I}}+\sigma_{x y}^{\mathrm{II}} \\
& \left.\sigma_{x y}^{\mathrm{I}}=\left\langle\hat{\sigma}_{x y}^{\mathrm{I}}\right\rangle=-\left\langle\operatorname{Tr} \epsilon_{\mu \nu} \Pi_{\mu} G^{+} \Pi_{\nu} G^{-}\right)\right\rangle_{\mathrm{imp}} \\
& \sigma_{x y}^{\mathrm{II}}=\left\langle\hat{\sigma}_{x y}^{\mathrm{I}}\right\rangle=i\left\langle\operatorname{Tr} \epsilon_{\mu \nu} r_{\mu} \Pi_{\nu}\left(G^{+}-G^{-}\right)\right\rangle_{\mathrm{imp}}
\end{aligned}
$$

In the above formula, $r_{\mu}$ denotes the position operator and $\Pi_{\mu}$ denotes the gauge invariant momentum operator. $G^{+}$and $G^{-}$are the advance and retarded one particle Green's functions respectively. $\langle\ldots\rangle_{\mathrm{imp}}$ denotes impurity average whereas $\langle\ldots\rangle_{\text {imp }}$ denotes both quantum mechanical trace and impurity average. The second term in the Lagrangian is called a topological term since for any smooth field configurations,

$$
\int d^{2} r \operatorname{Tr} \epsilon^{\mu \nu} Q \partial_{\mu} Q \partial_{\nu} Q=16 \pi i n
$$

where $n$ is an integer, called the Pontrjagin index. Different $n$ label different topological sectors with a given net instanton number. The presence of the topological term violates both time reversal and parity, and gives a handness to the two dimensional plane. Such a term is not allowed on symmetry grounds for the random flux problem with no net averaged magnetic field.

$\sigma_{x y}^{\text {II }}$ is a one body operator that measures the magnetization at the fermi energy, it receives only contribution from the edge states [14]15]. In the random field problem, its average vanishes, but its correlation due to the fluctuating magnetic domains is long ranged. To see this, we notice that its contribution to the topological term arises in a cumulant expansion of

$$
\begin{array}{r}
\left\langle\exp \frac{1}{8} \int d^{2} r \hat{\sigma}_{x y}^{\mathrm{II}}(r) \rho(r)\right\rangle=1+\frac{1}{8} \int d^{2} r\left\langle\hat{\sigma}_{x y}^{\mathrm{II}}(r)\right\rangle \rho(r)+ \\
\frac{1}{128} \int d^{2} r d^{2} r^{\prime}\left\langle\hat{\sigma}_{x y}^{\mathrm{II}}(r) \hat{\sigma}_{x y}^{\mathrm{II}}\left(r^{\prime}\right)\right\rangle \rho(r) \rho\left(r^{\prime}\right)+\ldots
\end{array}
$$

where $\rho(r)=\operatorname{Tr} \epsilon^{\mu \nu} Q \partial_{\mu} Q \partial_{\nu} Q$ is the local Pontrjagin density. In the presence of an uniform magnetic field, the second term in nonzero, reexponentialting it gives the topological term in the Lagrangian of equation (2). In this case, one has to reorganize the cumulant expansion into an expansion in $\hat{\sigma}_{x y}^{\mathrm{II}}-\left\langle\hat{\sigma}_{x y}^{\mathrm{II}}\right\rangle$. It can be easily shown that the correlation function of this operator is short ranged, therefore keeping higher order terms in the cumulant expansion would only give rise to irrelevant higher derivative terms. In the case of random flux with no net average, the second term in equation (5) vanish identically. One is left to calculate the two point correlation function of local $\hat{\sigma}$ operators. This correlation function can be calculated using a Ward identity relating it to the current current correlation function. Define the local current operator to be $J_{\mu}(r)=i \Pi_{\mu}(r)\left(G^{+}-G^{-}\right)$. For the purpose of calculating DC transport properties one can assume that the current is transverse, i.e $\partial_{\mu} J_{\mu}=0$. Furthermore let us assume that the current operator can be expanded in terms of its moments. Under these two assumptions,

$$
J_{\mu}(q)=-\frac{i}{2} \epsilon_{\mu \nu} q_{\nu} \hat{\sigma}_{x y}^{\mathrm{II}}(q)+o\left(q^{2}\right)
$$

This is nothing but the familiar identity $\vec{J}=\frac{1}{2} \vec{\nabla} \times \vec{M}$ in electromagnetism, expressing current in terms of the magnetic moment, which in our case is just $\sigma_{x y}^{\mathrm{II}}$. Since this is an operator identity, it can be used to derive the following identity between the correlation functions:

$$
\left\langle J_{\mu}(q) J_{\nu}(-q)\right\rangle=\frac{1}{4}\left(\delta_{\mu \nu} q^{2}-q_{\mu} q_{\nu}\right)\left\langle\hat{\sigma}_{x y}^{\mathrm{II}}(q) \hat{\sigma}_{x y}^{\mathrm{II}}(-q)\right\rangle+o\left(q^{4}\right)
$$

In the limit of $q \rightarrow 0$, the transverse current current correlation of the left hand side approaches $\sigma_{x x}$, therefore we obtain

$$
\left\langle\hat{\sigma}_{x y}^{\mathrm{II}}(q) \hat{\sigma}_{x y}^{\mathrm{II}}(-q)\right\rangle=\frac{4 \sigma_{x x}}{q^{2}}
$$

Since $\sigma_{x y}^{\mathrm{I}}$ is already a two body operator, its correlation function enters higher order cumulant expansion which will not be considered here. Inserting (8) into equation (5) and reexponentialte the third term, one obtains the following expression for the field theory action of the random flux model: 


$$
\mathcal{S}=\frac{\sigma_{x x}}{8} \int d^{2} r \operatorname{Tr}\left(\partial_{\mu} Q\right)^{2}-\frac{\sigma_{x x}}{32} \int \frac{d^{2} q}{(2 \pi)^{2}} q^{-2} \rho(q) \rho(-q)
$$

Thus we see that while there is no topological term in the action for the random flux problem, there is a term describing the long ranged interaction of the topological density.

The physical meaning of this nonlocal interaction term becomes transparent if one introduces an additional boson field to decouple the nonlocal term. The action becomes local after this decoupling:

$$
\mathcal{L}=\frac{\sigma_{x x}}{8} \operatorname{Tr}\left(\partial_{\mu} Q\right)^{2}+\frac{8}{\sigma_{x x}}\left(\partial_{\mu} \phi\right)^{2}+\phi(r) \rho(r)
$$

The above equation is the central result of this paper, we propose this action to be the effective field theory model describing the random flux problem. The last term in the above equation is reminiscent of the topological term, except that the constant topological angle is now a space dependent scalar field, and there is a gradient term for the scalar field. The gradient term keeps $\phi$ locally constant. The locally constant $\phi$ field can be viewed as the topological angle in a given magnetic domain in the random flux problem. Another physical interpretation can be obtained when we use the fermionic representation of the $\phi$ field. According to the bosonization rules, one scalar field can be represented by a Dirac fermi field, with the gradient term $\left(\partial_{\mu} \phi(r)\right)^{2}$ replaced by the Dirac action $\bar{\psi} \gamma^{\mu} \partial_{\mu} \psi$. The equation of motion for the bose field $\phi$ is

$$
\partial_{\mu}\left(\frac{16}{\sigma_{x x}} \partial_{\mu} \phi\right)=\rho
$$

The fermionized version of this equation is

$$
\partial_{\mu}\left(\frac{16 \sqrt{\pi}}{\sigma_{x x}} \bar{\psi} \gamma_{5} \gamma^{\mu} \psi\right)=\rho
$$

This is the famous chiral anomaly equation that arises in the resolution of the $U(1)$ problem in the quantum chromodynamics 16]. It is also similar to a model of holes moving in antiferromagnet background in $1+1$ dimensions [17]. The spin degrees of freedom is described by the $O(3)$ nonlinear sigma model whereas the massless fermions describes the holes in the antiferromagnetic background. These two degrees of freedom are coupled exactly through the anomaly equation (12). At the classical level, the two chiral components of the Dirac fermion are decoupled since the mass term is absent. However, this symmetry is broken at the quantum level by the presence of instantons. In the topologically trivial sector, the integrated Pontrjagin density vanishes and the chiral charge is conserved. The presence of instantons violates the conservation of the chiral charge.

Physically, one can view the two chiral components of the Dirac fermion as the gapless edge states of the fluctuating magnetic domains [18]. The right chiral fermion can be associated with the magnetic domain in the $+z$ direction, and the left chiral fermion can be associated with the magnetic domain in the opposite direction. One can visualize the world line of the two chiral fermions as the current loops of the edge states. The edge states can be extended over a distance far larger than the localization length, thus they appear to be gapless at the classical level. However, there is tunneling between the two chiral components, where the tunneling event in our formulation is associated with the instanton, and the tunneling rate given by the instanton action. A crucial question is whether the chiral anomaly (11) and (12) gives an effective mass for the Dirac fermion or if the fermions remain massless even in the presence of instantons. Formulated in another way, the question is whether the scattering between the two different types of edge states is relevant or irrelevant.

One way to answer this question is to integrate out the $Q$ fields in the nonlinear sigma model (10) and obtain an effective theory for the boson field $\phi$. One notices that there is a symmetry in the problem: if one shifts the $\phi$ field by a constant $\theta$, the gradient term remain unchanged. One picks up a contribution

$$
\theta \int d^{2} r \rho(r)=16 \pi i \theta n
$$

from the coupling of the $\phi$ field to the topological density. The above equality holds because of the quantization of the topological charge (4). For $\theta=\frac{1}{8}$, this shift contributes a factor of $e^{i 2 \pi n}=1$ to the action, thus $\phi(r) \rightarrow \phi(r)+\frac{1}{8}$ is an exact symmetry of the problem that must be respected by the effective Hamiltonian in $\phi$. A mass term for the $\phi$ field, $m^{2} \phi^{2}$ for example, is forbidden by this symmetry. Because of this symmetry, the effective action for $\phi$ can be expanded in a Fourier series,

$$
\mathcal{L}_{\text {eff }}=\frac{8}{\sigma_{x x}}\left(\partial_{\mu} \phi\right)^{2}-2 y \cos (16 \pi \phi)+\ldots
$$

The higher harmonics term have higher scaling dimensions compared to the lowest harmonic term, and they can be neglected in the analysis of critical properties. Thus, general symmetry considerations severely restrict the low energy effective action for the $\phi$ field to be the sine-Gordon model. At this level, $y$ is an unknown parameter. While this effective action for the $\phi$ field is obtained from general symmetry considerations, it can also be derived explicitly from a dilute instanton gas approximation. It is known that the nonlinear sigma model for the $Q$ matrices supports instanton solutions that survives the replica limit $m \rightarrow 0$ [14. They make a contribution of $y=D \exp \left(-4 \pi \sigma_{x x}\right)$ to the action, where the exponent is the classical action of an instanton and $D$ is the fluctuation determinant around the classical instanton. Assuming that these instantons can be treated as independent variables, one obtains the following for the effective action of the $\phi$ field 


$$
\begin{aligned}
e^{-S_{\text {eff }}[\phi]}= & \exp \left[\frac{1}{2}\left(\frac{16}{\sigma_{x x}}\right) \int d^{2} x\left(\partial_{\mu} \phi\right)^{2}\right. \\
& +\sum_{n_{+}, n_{-}} \frac{y^{n_{+}}}{n_{+} !} \frac{y^{n_{-}}}{n_{-} !} \int d x_{1} \cdots d x_{n_{+}} e^{16 \pi i \sum \phi\left(x_{i}\right)} \\
& \left.\times \int d \bar{x}_{1} \cdots d \bar{x}_{n_{-}} e^{-16 \pi i \sum \phi\left(\bar{x}_{i}\right)}\right]
\end{aligned}
$$

the sums and integrals can be done explicitly to give the effective action (14).

The effective action (14) is the sine-Gordon model for scalar field, and has a well known Kosterlitz-Thouless phase transition. After a rescaling of the field $\phi$ one obtains

$$
\mathcal{L}_{\text {eff }}=\frac{1}{2}\left(\partial_{\mu} \phi\right)^{2}-2 y \cos \left(4 \pi \sqrt{\sigma_{x x}} \phi\right)
$$

this is identical to the effective Lagrangian for the $X Y$ model [19] provided one makes the identification of $\sigma_{x x}$ with the inverse temperature $\beta=1 / k T$ and $y$ with the fugacity of the $X Y$ vortices. The Kosterlitz-Thouless transition occurs at $\sigma_{x x}^{c}=2 / \pi$. The conductance $\sigma_{x x}$ which enters the effective field theory is the "bare" conductance calculated within the self-consistent Born approximation. $\sigma_{x x}^{c}$ divides the regime where the cosine term is relevant from the one in which the cosine term is irrelevant. For $\sigma_{x x}<\sigma_{x x}^{c}$, one is in the high temperature phase where instantons form a free plasma and the system is disordered with a finite mass gap. However, for $\sigma_{x x}>\sigma_{x x}^{c}$, one is in the low temperature phase in which the instantons form tightly bound pairs. There is line of fixed points with power law correlations. The massive phase is identified with the localized phase in which the scattering between the two different chiral edge states are relevant, whereas the massless phase is identified with the phase where extended states exists and the scattering between the edge states is irrelevant. The current is carried by these gapless edge states and they are responsible for the finite continuously varying conductance in this regime. This picture is perfectly consistent with the recent numerical results of ref. [2, 13, 4], where it was suggested that a line of fixed points might exist in a finite region around the center of the band. The conductance was found to be finite and continuously varying in this energy range.

However, a word of caution concerning the dilute instanton gas approximation is in order here. In a model where the massless fermions are coupled to the gauge fields of the abelian Higgs model [20], the dilute instanton gas approximation is valid because the instanton has a well defined size. Consequently the existence of the Kosterlitz-Thouless phase transition is on a firm ground. For the case of massless fermions coupled to nonlinear sigma model, the instantons have arbitrary size and therefore they can overlap with each other which makes dilute instanton approximation hard to justify. This question was studied extensively in the context of the $C P^{n-1}$ model [21 23]. In the large $n$ limit, Witten
21] has shown that the instanton disappear after quantum fluctuations are taken into account, and the $\phi$ field is always massive. However, it is now well established that this large $n$ result does not hold for $n \leq 2$. For the case of $n=2$, the $C P^{n-1}$ model is equivalent to the $O(3)$ nonlinear sigma model. From the analogy with quantum spins chains, it is known that this model has a second order phase transition when the topological angle is equal to $\pi$, whereas the large $n$ theory predicts a first order phase transition [22]. For the case of $n=1$, the $C P^{n-1}$ model is equivalent to the abelian Higgs model, where the instanton calculus is certainly correct. Based on this general arguments, Affleck 23] has argued that the large $n$ result does not hold for $n \leq 2$. It is thus plausible that the qualitative feature obtained by the instanton calculus is valid in this regime, and in particular, in the replica limit of $n \rightarrow 0$ that we are interested here.

In conclusion we have constructed an effective field theory of the localization problem in random magnetic flux. This field theory has the same degrees of freedom as the nonlinear sigma model of unitary ensemble, but there is a long ranged logarithmic interaction of the topological density. This model can be reformulated in terms of a scalar field coupling to the topological density or equivalently, a Dirac fermi field obeying a chiral anomaly equation. These additional degrees of freedom can be physically interpreted as the edge states of the different magnetic domains. Our field theory model describes the tunneling between the edge states of different chirality in terms of the instantons of the nonlinear sigma model. The resulting effective field theory for the scalar field is shown to be a sine Gordon model from general symmetry considerations and we argue that such a model has a Kosterlitz-Thouless phase transition from localized states to extended states with power law correlations. This picture is consistent with the recent numerical calculations of the random flux model in which a divergent localization length was found and a finite region of continuously varying conductance was identified. Our results have important consequences for the localizations physics by demonstrating the existence of a finite region of extended states in two dimensions, and deepen our understanding of the "Hall metal" phase around the even denominator filling fractions in the quantum Hall systems.

The authors would like to acknowledge interesting and stimulating discussions with J. Chalker, D.H. Lee, H. Levine, S. Libby, N. Read, R. Shankar and A. Tikofsky. Part of this work was carried out at the Aspen center for physics during the workshop on the quantum Hall effect.

[1] P. A. Lee and T. V. Ramakrishnan, Rev. Mod. Phys. 57, 287, (1985).

[2] V. Kalmeyer and S. C. Zhang, Phys. Rev. B46, 9889 
(1992).

[3] B. I. Halperin, P. A. Lee and N. Read, Phys. Rev. B47, 7312 (1993).

[4] V. Kalmeyer D. Wei, D. Arovas, and S. C. Zhang, "TwoDimensional Localization in the Presence of Random Flux and the Quantum Hall System at Even Denominator FIlling Fractions", Phys. Rev. B (in press, October 1993).

[5] Kalmeyer and R. B. Laughlin, Phys. Rev. Lett. 59, 2095 (1987); X. G. Wen, F. Wilczek and A. Zee, Phys. Rev. B39, 11413 (1989).

[6] N. Nagaosa and P. A. Lee, Phys. Rev. Lett. 64, 2450 (1990).

[7] C. Pryor and A. Zee, Phys. Rev. B46, 3116 (1992).

[8] B. L. Altshuler and L. B. Ioffe, Phys. Rev. Lett. 69, 2979 (1992).

[9] G. Gavazzi, J. M. Wheatley, and A. J. Schofield, Phys. Rev. B47, 15170 (1993).

[10] D. V. Khveschenko and S. V. Meshkov, Phys. Rev. B47, 12051 (1993).

[11] S. Hikami, Prog. Theor. Phys. 107, 213 (1992).

[12] T. Sugiyama and N. Nagaosa, Phys. Rev. Lett. 70, 1980 (1993).

[13] Y. Avishai, Y. Hatsugai, and M. Kohmoto, Phys. Rev. B47, 9561 (1993).

[14] H. Levine, S. B. Libby, and A. M. M. Pruisken, Phys. Rev. Lett. 51, 1915 (1983); Nucl. Phys. B240, 30 (1984); Nucl. Phys. B240, 49 (1984); Nucl. Phys. B240, 72 (1984); A. M. M. Pruisken in The Quantum Hall Effect, R. E. Prange and S. M. Girvin, eds. (Springer-Verlag, 1990).

[15] P. Středa, J. Phys. C 15, L717 (1982).

[16] For a review, see S. Coleman, Use of instantons, in Aspects of Symmetry (Cambridge, 1988).

[17] R. Shankar, Nucl. Phys. B330, 433 (1990).

[18] This is similar with the identification of the chiral fermions with the edge states made in the recent literature. Physically we are dealing the chiral fermions in two dimensional space, whereas the following literature studies the dynamics in $1+1$ dimensional space time. X. G. Wen, Phys. Rev. Lett. 64, 2206 (1990); D.H. Lee and X.G. Wen, Phys. Rev. Lett. 66, 1765 (1991); M. Stone, Ann. Phys. 207, 38 (1991).

[19] J. Kogut, Rev. Mod. Phys. 51, 659 (1979).

[20] C. G. Callan Jr., R. Dashen, and D. J. Gross, Phys. Rev. D16, 2526 (1977).

[21] E. Witten, Nucl. Phys. B149, 285 (1979).

[22] I. Affleck, Nucl. Phys. B305, 582 (1988).

[23] I. Affleck Phys. Rev. Lett. 66, 2429 (1991). 